\title{
Revision of loop colostomy under regional anaesthesia and sedation
}

\author{
Oriana $\mathrm{Ng}^{1}$, MBBS, MMed, Sze Ying Thong ${ }^{1}$, MBBS, MMed, Claramae Shulyn $\underline{\text { Chia }}^{2}$, MBBS, FRCS, \\ Melissa Ching Ching ${\underline{T e 0^{2}}}^{2}$ MBBS, MRCS
}

\begin{abstract}
Patients presenting for emergency abdominal procedures often have medical issues that cause both general anaesthesia and central neuraxial blockade to pose significant risks. Regional anaesthetic techniques are often used adjunctively for abdominal procedures under general anaesthesia, but there is limited published data on procedures done under peripheral nerve or plexus blocks. We herein report the case of a patient with recent pulmonary embolism and supraventricular tachycardia who required colostomy refashioning. Ultrasonography-guided regional anaesthesia was administered using a combination of ilioinguinal-iliohypogastric, rectus sheath and transversus abdominis plane blocks. This was supplemented with propofol and dexmedetomidine sedation as well as intermittent fentanyl and ketamine boluses to cover for visceral stimulation. We discuss the anatomical rationale for the choice of blocks and compare the anaesthetic conduct with similar cases that were previously reported.
\end{abstract}

Keywords: abdominal wall surgery, colostomy, ilioinguinal-iliohypogastric, rectus sheath and transversus abdominis plane blocks, ultrasonography-guided regional anaesthesia

\section{INTRODUCTION}

The use of central neuraxial blockade (CNB) alone for abdominal surgery has been reported in the literature. ${ }^{(1,2)}$ However, patients who present for emergency abdominal procedures often have medical issues that cause both general anaesthesia (GA) and CNB to pose significant risks. It is also theoretically feasible to use specific trunk blocks alone, but there are a limited number of reports on the use of this technique for abdominal surgery. We herein report the case of a patient who underwent colostomy refashioning under ultrasonography-guided truncal regional anaesthesia (RA).

\section{CASE REPORT}

A 72-year-old Middle Eastern man was admitted electively for laparoscopic colostomy creation, which was uneventful. He weighed $68 \mathrm{~kg}$, had known hypertension, hyperlipidaemia and diabetes mellitus, and was also recently diagnosed with sigmoid colon adenocarcinoma with metastases to the vertebrae and liver. The patient was commenced on radiotherapy on postoperative day (POD) 6. Chemotherapy with cituximab was started after the completion of ten cycles of radiotherapy on POD 15. He was also started on a fentanyl infusion $50 \mu \mathrm{g} / \mathrm{hr}$ because of continuous pain from the metastases.

The patient's postoperative course was complicated on POD 9 by embolism in the right main pulmonary artery, for which he was started on low-molecular-weight heparin enoxaparin $1 \mathrm{mg} / \mathrm{kg}$ twice daily. He subsequently complained of significant abdominal distension with minimal passage of gas from the stoma. On clinical examination, an oedematous stoma was seen. A large-bore Foley catheter was inserted into the stoma, but it failed to relieve the distension. Refashioning of the stoma was thus scheduled to relieve the bowel obstruction on POD 13. In anticipation of the perioperative discontinuation of anticoagulants, an inferior vena cava filter was inserted when the patient was in the radiology suite just prior to transport to the operating theatre. At this point, his treatment dose of enoxaparin had been ceased for 17 hours.

Apart from the recent pulmonary embolism, use of anticoagulants and chronic opioid exposure, the patient's other active medical issues were transaminitis and hyperbilirubinaemia due to the progressive metastatic disease, as well as anaemia (haemoglobin level $8.2 \mathrm{~g} / \mathrm{dL}$ ) and coagulopathy (international normalised ratio 1.45).

During pre-induction, he was found to be experiencing supraventricular tachycardia, with a heart rate of $160 \mathrm{bpm}$ that was not reversed by vagal manoeuvres. Supraventricular tachycardia reverted to sinus tachycardia at $110 \mathrm{bpm}$ after a total of $100 \mathrm{mg}$ of esmolol and $5 \mathrm{mg}$ of labetalol was administered. Room air saturation was at $88 \%$ and his blood pressure was within an acceptable range. Due to the absence of any obviously reversible cause for the tachyarrhythmia, an urgent on-table cardiology referral was made and bedside echocardiography was performed. The study revealed no thrombus, regional wall motion abnormalities or right-sided heart failure.

At this point, the anaesthesia, surgery and cardiology teams discussed the need to proceed with colostomy refashioning and decided to proceed with the surgery, as the patient was scheduled for urgent chemotherapy to control the metastases; the risk of perioperative bleeding and infection would be greatly elevated during chemotherapy. RA using a combination of truncal blocks and systemic analgesia was chosen, as it would cause less haemodynamic disturbance compared with GA.

After standard monitoring was applied, the patient was premedicated with a $50 \mathrm{mcg}$ bolus of intravenous fentanyl. Sedation was initiated using a target-controlled infusion of

${ }^{1}$ Department of Anaesthesiology, Singapore General Hospital, ${ }^{2}$ Division of Surgical Oncology, National Cancer Centre Singapore, Singapore 
propofol (Schnider model) at an effect site concentration of $0.7 \mathrm{mcg} / \mathrm{mL}$. Oxygen was administered at $6 \mathrm{~L} / \mathrm{min}$ via a Hudson face mask to maintain saturation of $100 \%$. RA was administered under sterile conditions using a SonoSite M-Turbo machine to provide real-time ultrasonography guidance. A 50-mm B Braun Stimuplex peripheral block needle was used. The toxic dose of local anaesthesia was precalculated to be $476 \mathrm{mg}$ of lignocaine if used with adrenaline. The following blocks were given on the left side of the abdomen: (a) ilioinguinal-iliohypogastric block with $10 \mathrm{~mL}$ of lignocaine $1.5 \%$ with adrenaline 1:200,000; (b) transverse abdominis plane (TAP) block using a midaxillary approach with a mixture of $5 \mathrm{~mL}$ of ropivacaine $0.5 \%$ and $10 \mathrm{~mL}$ of lignocaine 1.5\% with adrenaline 1:200,000; and (c) rectus sheath block with a mixture of $15 \mathrm{~mL}$ of ropivacine $0.5 \%$ and $5 \mathrm{~mL}$ of lignocaine $1 \%$. The total dose of local anaesthesia received was $350 \mathrm{mg}$ of lignocaine and $100 \mathrm{mg}$ of ropivacaine. The time taken for performance of all three blocks was 25 minutes.

Target-controlled infusion propofol was increased to a target effect-site concentration of $1.2 \mathrm{mcg} / \mathrm{mL}$ for the commencement of surgery. In addition, the patient was given a further bolus of 50 mcg of fentanyl and $10 \mathrm{mg}$ of ketamine in anticipation of bowel manipulation. As the patient was saturating poorly at baseline, a dexmedetomidine infusion starting at $0.5 \mathrm{mcg} / \mathrm{kg} / \mathrm{hr}$ was started to optimise analgesia without significantly increasing the risk of respiratory depression. Knife-to-skin occurred five minutes after completion of the last block. Surgery proceeded uneventfully and lasted 20 minutes. No supplementary or rescue local anaesthetic was needed. During this time, the maximum target effect-site concentration of propofol was $1.5 \mathrm{mcg} / \mathrm{mL}$ and the maximum infusion rate of dexmedetomidine was $0.9 \mathrm{mcg} / \mathrm{kg} / \mathrm{hr}$. Surgical stimulus was pre-emptively treated by boluses of ketamine that were administered $10 \mathrm{mg}$ at a time, up to a total of $120 \mathrm{mg}$ throughout the procedure. A Richmond Agitation-Sedation Scale score of -2 to -4 was achieved with this technique. The patient remained haemodynamically stable throughout.

Postoperatively, the patient was monitored in the recovery area for 30 minutes, during which he required no additional analgesia. He was subsequently discharged to the highdependency unit. A review on POD 1 demonstrated no immediate complications from either the surgery or conduct of anaesthesia.

\section{DISCUSSION}

In this case study, the selection of an RA technique for the patient arose from the need to avoid potential haemodynamic instability and postoperative respiratory complications, in view of his multiple comorbidities at the time of the procedure. CNB was also contraindicated in view of coagulopathy and recent administration of low-molecular-weight heparin. With regard to anticoagulation, current guidelines recommend similar precautions for neuraxial techniques and peripheral blocks. ${ }^{(3)}$ However, it stands to reason that bleeding complications in a peripheral and superficial compartment would likely be less catastrophic than bleeding in a central neuraxial or deep plexus compartment. In this particular patient, the benefits of avoiding both GA and CNB outweighed any potential risks of a truncal block.
Regional techniques used in the trunk region for postoperative pain relief, especially TAP blocks, are frequently used adjunctively for abdominal procedures performed on patients under GA. ${ }^{(4)}$ However, there is a paucity of reports detailing the use of these techniques as the sole anaesthetic with or without sedation and analgesia. The use of a bilateral rectus sheath block with $10 \mathrm{~mL}$ of $0.5 \%$ bupivacaine and $5 \mathrm{~mL}$ of $1 \%$ lignocaine with adrenaline on each side has been described in the mesh repair of a paraumbilical hernia in a patient with significant cardiac risk factors. The patient had received $0.5 \mathrm{mg}$ of midazolam and $10 \mathrm{mg}$ of ketamine prior to the block and required only a further $25 \mathrm{mcg}$ of fentanyl, $0.5 \mathrm{mg}$ of midazolam and $10 \mathrm{mg}$ of ketamine during bowel handling. No other anaesthesia was required during the 90 -minute surgery. ${ }^{(5)}$ Bilateral rectus sheath block has also been used for infraumbilical hernia repair in a morbidly obese patient with significant cardiac risk factors. The patient received $15 \mathrm{~mL}$ of $1 \%$ lignocaine and $10 \mathrm{~mL}$ of $0.5 \%$ bupivacaine bilaterally under ultrasonography guidance and tolerated surgery with minimal further sedation. ${ }^{(6)}$

Jensen et al used bilateral dual TAP blocks in an American Society of Anesthesiology (ASA) class IV patient undergoing revision of abdominal wall defects following an emergency laparotomy. A total of $40 \mathrm{~mL}$ of lignocaine $1 \%$ with adrenaline was administered at two points on each side of the abdomen, resulting in decreased sensation from dermatomes T6 to T12. The patient received oxygen via face mask and $2 \mathrm{mg}$ of midazolam for the duration of the surgery, which was uneventful. ${ }^{(7)}$ Mishra et al similarly performed bilateral subcostal TAP blocks using a single injection on either side for an ASA class IV patient undergoing emergency laparotomy of a perforated ileum. A total of $40 \mathrm{~mL}$ of $0.25 \%$ bupivacaine, $40 \mathrm{~mL}$ of $1 \%$ lignocaine and $0.2 \mathrm{mg}$ of adrenaline was administered. The patient was sedated with an intravenous infusion of dexmedetomidine and oxygen was delivered via face mask. The authors commented that although the abdominal wall incision was painless, the patient complained of pain during manipulation of the intestine. This was mitigated by the dexmedetomidine infusion. ${ }^{(8)}$

The combination of trunk blocks chosen in this case was based on knowledge of the anatomy of the abdominal wall in relation to the site of surgery, i.e. a colostomy in the left iliac fossa region. This area is innervated by the anterior and lateral cutaneous branches of the intercostal nerves corresponding to dermatomes T10-T12, with presumed variable contribution from the nerves supplying the dermatomes immediately above and below this band. The TAP block targeted dermatomes T10-12 on the anterolateral aspect of the abdominal wall. An ilioinguinal block was administered to incorporate coverage of the first lumbar nerve supply, while the rectus sheath block was given to cover the more medial portion of the anterior abdominal wall (Fig. 1).

The need for supplemental analgesia was inevitable given that the trunk blocks, unlike CNB, would cover cutaneous sensation but not visceral stimulation from manipulation of the colon that was being refashioned. For the same reason, gentle surgical manipulation was required. 

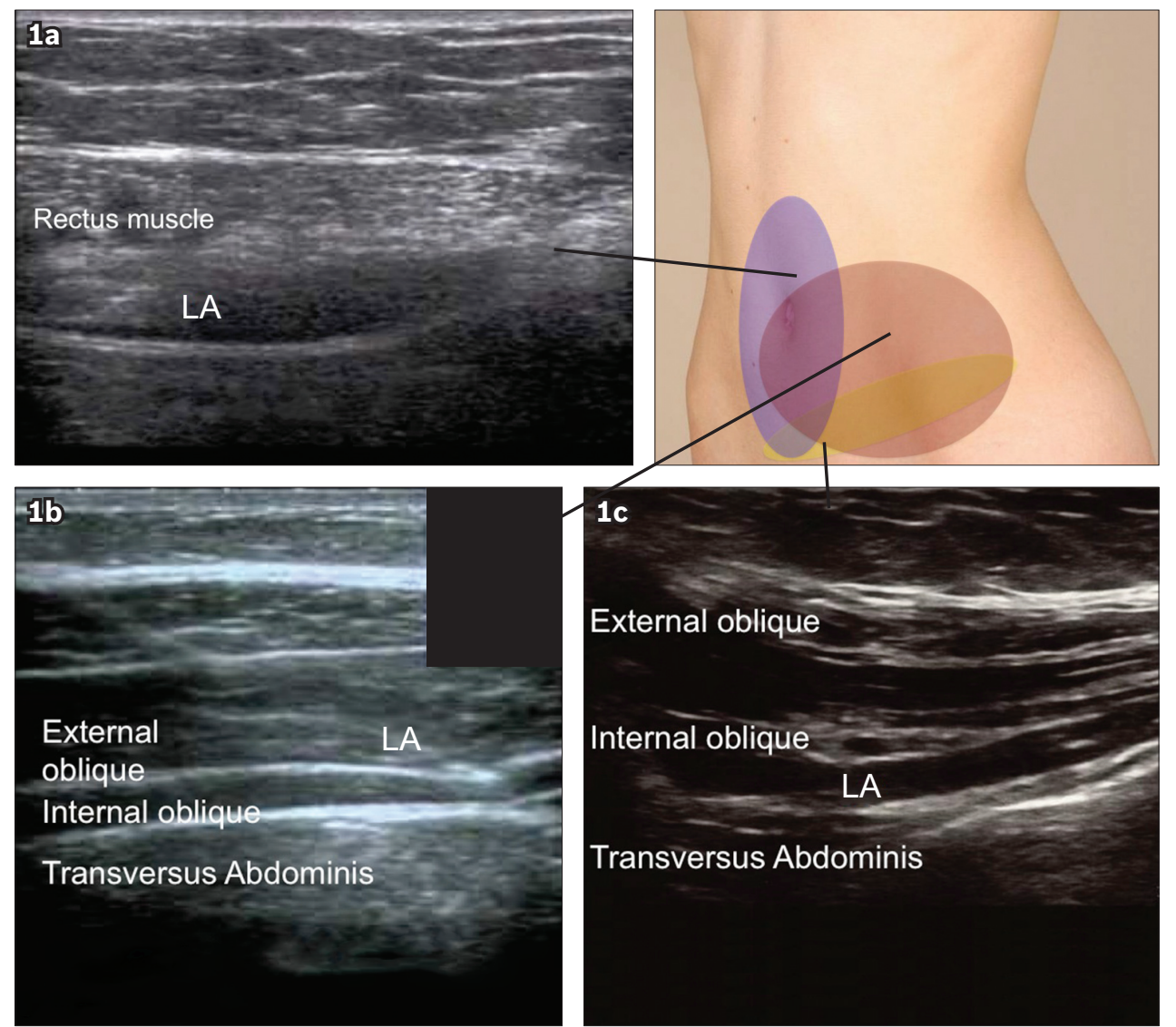

Fig. 1 Regions of the anterior abdominal wall affected by the different blocks administered. (a) US image of a rectus sheath block shows local anaesthesia being deposited between the rectus muscle and the posterior rectus sheath; (b) US image of a transversus abdominis plane block shows local anaesthesia being deposited between the internal oblique and transversus abdominis muscles; (c) US image of an ilioinguinal-iliohypogastric block shows local anaesthesia being deposited between the internal oblique and transversus abdominis muscles.

The total local dose of anaesthesia received was $350 \mathrm{mg}$ of lignocaine and $100 \mathrm{mg}$ of ropivacaine, which translates to approximately $5 \mathrm{mg} / \mathrm{kg}$ and $1.4 \mathrm{mg} / \mathrm{kg}$ of lignocaine and ropivacaine, respectively. Combinations of local anaesthetics are considered to have an additive effect. The maximum recommended dose of lignocaine is $3 \mathrm{mg} / \mathrm{kg}$ without adrenaline and $7 \mathrm{mg} / \mathrm{kg}$ with adrenaline, while the maximum for ropivacaine is $3-4 \mathrm{mg} / \mathrm{kg}$ with or without adrenaline, although some studies have reported using $5 \mathrm{mg} / \mathrm{kg}$ of ropivacaine for local infiltration or plexus blocks without achieving toxic manifestations or plasma concentrations. ${ }^{(9-11)}$ By extension, the amount of local anaesthesia used in our patient was within acceptable limits, although standard safety measures and monitoring were still required.

In conclusion, trunk blocks with supplementary analgesia and sedation are a feasible anaesthetic option for patients undergoing minor abdominal procedures and in whom GA and CNB are risky or contraindicated.

\section{REFERENCES}

1. Savas JF, Litwack R, Davis K, Miller TA. Regional anesthesia as an alternative to general anesthesia for abdominal surgery in patients with severe pulmonary impairment. Am J Surg 2004; 188:603-5.
2. England RJ, Blues C, Amin SN. Reversal of loop ileostomy under spinal anaesthesia. Int J Colorectal Dis 2005; 20:349-52.

3. Horlocker TT, Wedel DJ, Rowlingson JC, et al. Regional anesthesia in the patient receiving antithrombotic or thrombolytic therapy: American Society of Regional Anesthesia and Pain Medicine Evidence-Based Guidelines (Third Edition). Reg Anesth Pain Med 2010; 35:64-101.

4. Abdallah FW, Chan VW, Brull R. Transversus abdominis plane block: a systematic review. Reg Anesth Pain Med 2012; 37:193-209.

5. Phua DS, Phoo JW, Koay CK. The ultrasound-guided rectus sheath block as an anaesthetic in adult paraumbilical hernia repair. Anaesth Intensive Care 2009; 37:499-500.

6. Quek K. A case report of an open infra-umbilical hernia repair under bilateral rectus sheath blocks (Abstract). ANZCA ASM 2012.

7. Jensen K, Baek N, Jensen JT, Brglum J. Bilateral dual transversus abdominis plane block providing surgical anaesthesia for abdominal wall surgery. Anaesthesia 2013; 68:106-8.

8. Mishra L, Pani N, Mishra D, Patel N. Bilateral transversus abdominis plane block as a sole anesthetic technique in emergency surgery for perforative peritonitis in a high risk patient. J Anaesthesiol Clin Pharmacol 2013; 29:540-2.

9. Beecroft C, Davies G. Systemic toxic effects of local anaesthetics. Anaesth Intens Care Med 2010; 11:98-100.

10. Pettersson N, Emanuelsson BM, Reventlid H, Hahn RG. High-dose ropivacaine wound infiltration for pain relief after inguinal hernia repair. Reg Anesth Pain Med 1998; 23:189-96.

11. Salonen MH, Haasio J, Bachmann M, Xu M, Rosenberg PH. Evaluation of efficacy and plasma concentrations of ropivacaine in continuous axillary brachial plexus block: high dose for surgical anesthesia and low dose for postoperative analgesia. Reg Anesth Pain Med 2000; 25:47-51. 